Cahiers de Narratologie

Analyse et théorie narratives

13 | 2006

Nouvelles approches de l'intertextualité

\title{
Le palimpseste de l'Histoire
}

\section{Nathalie Piégay-Gros}

\section{CpenEdition}

Journals

Édition électronique

URL : http://journals.openedition.org/narratologie/344

DOI : 10.4000/narratologie.344

ISSN : 1765-307X

Éditeur

LIRCES

\section{Référence électronique}

Nathalie Piégay-Gros, «Le palimpseste de l'Histoire », Cahiers de Narratologie [En ligne], 13 | 2006, mis en ligne le 01 septembre 2006, consulté le 15 novembre 2019. URL : http://journals.openedition.org/ narratologie/344 ; DOI : 10.4000/narratologie.344

Ce document a été généré automatiquement le 15 novembre 2019.

\section{(c) (i) (9)}

Cahiers de Narratologie - Analyse et théorie narratives est mis à disposition selon les termes de la licence Creative Commons Attribution - Pas d'Utilisation Commerciale - Pas de Modification 4.0 International. 


\title{
Le palimpseste de l'Histoire
}

\author{
Nathalie Piégay-Gros
}

1 Deux discours peuvent être distingués dans le commentaire fourni qu'Aragon a développé sur La Semaine sainte : il a souligné l'improvisation du roman, dont le style, à bien des égards, garde la trace; il a aussi noté l'importance des recherches documentaires qui lui ont été nécessaires pour écrire la fuite de Louis XVIII et le retour de Napoléon, débarqué de l'Ile d'Elbe en mars $1815^{1}$. Ces deux caractérisations de l'écriture s'opposent nettement: d'une part, la valorisation de l'invention et de l'écriture rapide, le pouvoir des mots et la confiance en la narration, imprévisible mais toujours désirée et à l'égard de laquelle l'auteur-lecteur manifeste une constante disponibilité ; d'autre part, l'exactitude historique, le souci réaliste, l'érudition. Si, dans le mouvement même de l'invention, qui enchaîne les mots et les surprises, les trouvailles et les épisodes, les lectures antérieures peuvent toujours resurgir, elles n'ont pas la même finalité que celles qui servent l'exactitude de la représentation ; elles sont en amont même de l'écriture le sous-bassement du roman et nourrissent l'imagination. C'est la liaison entre improvisation et savoir, écriture conçue comme une lecture et écriture nourrie de lectures, diversement drainées par le flot de la phrase, que je voudrais étudier. Non que le roman dissimule la documentation, qu'il assimilerait au point de la faire disparaitre de la surface du texte; souvent, au contraire, les textes lus sont évoqués de manière explicite jouant de la tension constitutive entre histoire et roman ${ }^{2}$; mais l'intertexte, dans La Semaine sainte, ne se limite pas aux lectures faites dans un souci de réalisme; il surgit aussi dans les trous creusés que les intrusions d'auteur creusent dans le tissu de l'histoire et du roman. L'intertextualité nous confronte donc à l'Autre de la fiction, qu'il s'agisse du discours de l'histoire ou de celui de l'auteur. Mais elle pose aussi la question des limites de l'intertextualité ; à mesure que nous pénétrerons les arcanes du texte, qui conduisent de l'intertextualité explicite aux formes implicites de récriture, la finalité et la légitimité de la notion devront être considérées ; car toute analyse qui fait appel à cette notion implique une critique de la lecture, pour autant que l'on n'en fasse pas un cache misère interprétatif ni un substitut facile de la critique des sources.

L'intertextualité explicite, entre hétérogénéité et cohérence 
2 Toute étude de l'intertextualité dans La Semaine sainte doit prendre en compte l'usage résolument non systématique qui est fait des textes convoqués par le roman. Travaillant tous azimuts, l'intertexte joue de la tension entre fiction et histoire, sans jamais inféoder la première à la seconde, mais sans, pour autant, dissimuler la difficile liaison de la vérité et du mensonge, de l'improvisation et de l'érudition. L'hétérogénéité, si importante dans ce roman qui laisse éclater la fascination pour la bigarrure (celle des costumes en particulier), le goût du désordre, de l'anarchie est naturellement favorable à l'intertextualité explicite. C'est que, pour Aragon, le roman est « bordel " ${ }^{3}$, assemblage hétéroclite d'éléments qui concourent à une cohérence d'un autre ordre. A cet égard, La Semaine sainte annonce La Mise à mort, Blanche ou l'oubli, et Théâtre/Roman, romans de la fracture, de la dissémination, de l'altérité apprivoisée. Mais cette dominante esthétique, si originale dans un roman historique, ne doit pas laisser penser que l'intertextualité tire toujours le texte du côté de l'hétérogène; Aragon la met aussi au service de la cohérence de la représentation.

3 Ainsi, la pratique de la citation, qui deviendra si importante dans les derniers romans d'Aragon, est présente dans La Semaine sainte plus que dans les romans du Monde réel ${ }^{4}$. Elle permet ainsi de consolider l'historicité de la représentation. C'est le cas des citations des Souffrances du jeune Werther, que convoque de manière sporadique mais insistante le personnage de Bernard ; profondément marqué par la lecture de Goethe, il trouve dans le roman une image de son désespoir amoureux et s'identifie si bien à son héros qu'il se suicide comme lui. L'historicité de la référence est importante, puisqu'elle permet de confirmer le « mal du siècle » qui frappe la génération des fils des soldats de l'Empire.

4 Mais les citations peuvent aussi, à l'inverse, souligner l'hétérogénéité du roman historique, dont l'hybridation est essentielle. C'est le cas, en particulier, lorsque les allusions ou citations affichent les textes qui ont précédé et autorisé l'invention : elles donnent alors accès au chantier de l'œuvre et rendent explicite la liaison indissoluble qu'il établit entre histoire et fiction, mensonge et vérité. Ainsi, dans le chapitre «Les graines de l'avenir", Aragon montre délibérément les textes qui lui ont permis d'évoquer la ville de Bamberg où se trouve le maréchal Berthier ; après avoir martelé qu'il n'a « jamais mis les pieds à Bamberg ", il mentionne le plan du théâtre qu'il en a, « qui est de 1912 », et sur lequel « il est marqué que le prix de la place dans la « Loge des Etrangers » est pour la comédie de deux marks cinquante pfennigs, trois marks cinquante pour l'opéra "; il renvoie également au Chat Mürr de E. T. A Hoffmann, et à L'Inspecteur des ruines d'Elsa Triolet ${ }^{5}$. Ces références sont alors une incitation puissante à l'investigation généalogique. L'érudition n'est plus cantonnée à l'antichambre du roman ; elle y pénètre, sur la pointe des pieds, mais non sans sourire. C'est encore le cas lorsqu'Aragon déplore le manque de travaux consacrés à un personnage pour lequel il manifeste beaucoup d'affection, Richelieu : "Il n'existe, à ma connaissance, dans notre pays, que deux ouvrages sur cet homme singulier, une monographie de la fin du XIX ${ }^{\mathrm{e}}$ siècle, et un livre de la même époque sur son action au congrès d'Aix-la-Chapelle ${ }^{6}$ ".

Ces citations mettent fin au bel isolement du roman historique, à distance du temps de l'écriture : elles font alors infraction au déroulement de la chronologie et métissent la fiction de discours autobiographique; c'est la juste séparation des genres qu'elles remettent en question. Le roman historique apparaît comme l'espace improbable dans lequel l'autobiographie peut venir se loger : « outre que ce souvenir, qu'y puis-je, c'est à voir Théodore Géricault dans son fourré, en contrebas, guettant les conjurés de Poix, 
qu'il m'est revenu, d'un coin obscurci de ma mémoire, une histoire que je n'ai même alors, au lendemain, jamais racontée. C'est que c'est à peine une histoire, seulement un tableau, une sensation passagère. J'avais vingt-deux ans, pas même, puisque c'était au printemps ou à la fin de l'hiver de 1919, près de Sarrebruck [...] »; la digression se clôt par deux vers de Feu de joie: «La beauté la seule vertu / Qui tende encore ses mains pures ... »; le narrateur souligne l'écart et le motive: "Mais qu'est-ce que tout cela vient faire là-dedans? Avec tout cela, j'ai perdu le fil de ce colloque de mars 1815, une part des choses dites, et j'y reviens plus égaré encore que Théodore Géricault, mousquetaire du $\operatorname{Roi}^{7}$ ".

6 Dans cette perspective, la longue autocitation des Communistes $^{8}$ loin de conforter la cohérence strictement fictionnelle, montre elle aussi comment le roman historique peut corriger l'histoire personnelle. Citer les Communistes au lieu de décrire la ferme où arrivent les fuyards royaux, c'est montrer les lieux communs aux deux romans; le commentaire que suscite cette autocitation accroît encore la part autobiographique de ce "roman, inachevé ${ }^{~}$, la fiction étant l'espace paradoxal du métatexte et du discours autobiographique.

7 L'intertextualité, comme le montrent les citations si différentes de Werther et de Feu de joie, à l'instar du roman dans son ensemble, est habitée par deux forces contradictoires, celle, centripète, de l'érudition, si sensible à l'exactitude des faits, au détail, d'autant plus présent que la diégèse est courte (une semaine) et la narration ample (plus de huit cents pages) et celle, centrifuge, de l'anarchie, du désordre, dont l'auteur dit qu'elle fait " craquer [s]on habit de tous les jours, l'habit de tous [s]es jours ${ }^{10}$ ».

La relation polémique à l'histoire

8 L'hétérogénéité que le texte laisse affleurer de manière plus discrète, en faisant l'économie de tout commentaire auctorial, ajoute aussi à cette ambivalence : la fiction s'autorise du discours historique, attestant ainsi son authenticité (effet de réel) mais ne le fait qu'en mentionnant le texte, si bien que le roman se donne à lire comme montage de textes préalables (effet de texte). La relation de l'algarade qui mit aux prises le duc de Berry avec un lancier, à Béthune est un exemple probant de cette ambivalence :

Cavaliers et piétons de la Maison étaient près d'un millier sur cet étroit espace, si bien que les volontaires, qui arrivaient et manquaient d'expérience, se crurent quatre mille. C'est dans ce tohu-bohu que, voyant sur l'un des chefs d'escadron la Croix de Saint-Louis, Monseigneur y vint droit: « De quel ordre avez-vous quitté vos cantonnements?»La suite est diversement racontée, personne sauf les cavaliers qui étaient près du Prince n'entendit les vraies paroles dont on a retenu, à peu près tous les historiens, ce que les volontaires royaux en racontèrent, lesquels étaient à bonne distance, et n'en entendirent pas mot [...] Il y a des gens qui prétendent qu'à cette minute un capitaine de lanciers reconnut dans un grenadier de La Rochejaquelein un de ses amis intimes et prononça de fort belles paroles, qu'on pouvait le tuer, mais qu'il ne frapperait pas un Français... ${ }^{11}$.

9 Aragon ne mentionne les discours des historiens, auxquels il feint d'adosser la scène, que pour corriger leurs approximations: le discours du roman est plus vrai (ou plus probable) que celui de l'histoire, que la fiction renvoie à ses incertitudes et ses aléas.

10 Les nombreux passages où sont relatés, sous forme de discours rapportés, les propos d'un personnage témoignent de la même tension entre souci de vérité historique et risque pris à l'authentification du discours. Aragon rétrocède les propos à leur locuteur, donnant corps et voix à un texte qui retrouve, dans le roman, son urgence et sa valeur dramatique. C'est le cas, à la fin du roman lorsque le personnage d'Alphonse de Prat, 
enfin identifié à Lamartine, prononce un long discours appelant à l'union des défenseurs de la liberté, fussent-ils royalistes, et des républicains, contre la tyrannie représentée par Napoléon ${ }^{12}$. Le texte présente les propos de Lamartine comme un discours direct, dont il souligne l'hétérogénéité en employant les caractères italiques ; il s'agit en fait de citations alors des Confidences. La nuance est importante: la transposition du discours n'est pas équivalente à une greffe. Si les deux retranscrivent, le discours transposé est du côté de la fabrique du texte (passage de l'oral à l'écrit), le discours cité du côté de sa restitution ou répétition (de l'écrit à l'écrit ${ }^{13}$ ). La même stratégie est employée pour relater le long discours de Fabvier sur le pas de la Bidassoa; l'italique semble ne souligner que le discours direct alors qu'il s'agit d'une longue citation ${ }^{14}$. Dans les deux cas, le roman s'approprie le discours historique : non sans paradoxe, la citation ne produit pas d'« effet de texte "; tout se passe au contraire comme si la médiation des sources historiennes était occultée.

11 Mais Aragon peut dissimuler plus avant la citation, en omettant de démarquer le passage qu'il emprunte; il prête à la rumeur ce qu'il tient de Mémoires qu'il cite exactement :

On raconte que le matin même de sa mort, un de ses lieutenants les plus fidèles, Duroc, avait dit dans un découragement prophétique : « Il nous fera tous tuer... pas un d'entre nous ne rentrera chez lui... » Et Junot qu'il avait fait duc, et rendu fou, en 1813, dans les éclairs de sa folie ne lui écrivait-il pas : «Moi qui vous aime avec l'adoration du sauvage pour le soleil, cette guerre éternelle qu'il faut faire pour vous, je n'en veux plus! je n'en veux plus! » C'était d'Aubigny qui lui avait raconté cela, qui le tenait du jeune Regnault de Saint-Jean-d'Angély ${ }^{15}$.

Ce qui est occulté, c'est l'emprunt aux Mémoires de Caulaincourt et à ceux de la duchesse d'Abrantès; l'imputation du discours à la rumeur («on»; «le jeune Regnault " puis d'Aubigny) déjoue la question de la source du discours. Alors même que le roman cite des fragments textuels, il feint de rapporter des paroles. Le discours, transposé ou rapporté, permet l'assimilation complète du discours historique dans la fiction.

13 Le personnage de Simon Richard fournit une autre preuve de ce statut paradoxal de l'érudition, qui, tout en servant l'exactitude de la représentation historique, favorise aussi l'invention au point même d'autoriser l'écart romanesque. On sait l'énigme qu'Aragon laisse planer sur l'identité de Simon Richard, alias Comte Olivier ${ }^{16}$; alors même qu'il a identifié la maîtresse de Tony de Reiset, et par conséquent son époux, Octave de Ségur, Aragon lui laisse son nom imaginaire de comte Olivier. La longue enquête sur la disparition de cet homme, engagé en Russie, puis suicidé par noyade, est évoquée par le roman; le père d'Octave/Olivier a mobilisé tous ses pouvoirs pour retrouver son fils disparu:

aux premiers jours de septembre il avait signalé à la police une maison où l'on entendait la nuit du bruit «comme si on y gardait quelqu'un ... » disait-il. Il avait espoir qu'on lui avait enlevé ce fils, qu'on le claustrait. Les hommes de Fouché avaient entouré la maison, c'était du côté de La Celle Saint-Cloud, dans les ténèbres : et quand on y avait fait irruption, c'était une boulangerie, le bruit était celui du geindre à pétrir ${ }^{17}$.

14 Pour cet épisode, Aragon puise aux mémoires de Fouché ${ }^{18}$, qu'il amplifie ; mais le but de cette anecdote historique exacte n'est pas de révéler l'identité du personnage romanesque, qui demeure cryptée. L'enquête de police à laquelle emprunte Aragon 
conforte ainsi la fiction, le discours de l'histoire cautionnant ici le pouvoir et la liberté de l'imagination, les errances et les hasards de l'invention.

On mesure, à la lecture de ces différents exemples, que l'on pourrait encore multiplier, combien les stratégies d'appropriation ou de détournement des textes lus sont variées : Aragon peut afficher ses sources ou les dissimuler. La mention des textes historiques auxquels il se réfère lui permet d'authentifier la fiction, sans jamais l'y inféoder ; mais il rompt aussi par là l'illusion référentielle et entretient une relation polémique avec le discours de l'histoire.

Si nous avançons sur les chemins glissants de l'intertextualité implicite, sans encore aborder les rivages très périlleux de l'intertextualité que rien ne signale, nous en arrivons à l'analyse des passages assez nombreux où seule l'identification de l'écrivain (par exemple Lamartine) derrière le personnage (Alphonse ou Monsieur de Prat), est un indice probant de l'intertextualité. Aragon pastiche ainsi les textes de Chateaubriand et Vigny ${ }^{19}$; ici, à l'évidence, c'est moins la perception d'une hétérogénéité qui conduit à la présomption d'un texte sous le texte que la mention d'un auteur. Comme si l'on déduisait, parce que l'on est dans un roman historique, du statut référentiel du personnage au caractère d'abord référentiel des propos que la fiction lui prête. Le pouvoir de l'histoire est grand, qui fait du discours du roman son débiteur.

L'analyse peut être poursuivie plus avant dans les arcanes de plus en plus secrets de l'intertexte. On touche alors aux textes non démarqués, qu'Aragon transforme pour inventer ${ }^{20}$. Nul indice d'hétérogénéité dans le roman, mais la présomption d'un savoir trouvé dans les discours antérieurs, qu'Aragon, nous le rappelions, a d'ailleurs pu signaler. Le jeu de la fiction et de l'histoire prend alors un tour différent: le roman n'authentifie pas nécessairement son discours, mais laisse au flot de la phrase charrier les décombres des textes lus parfois très précisément, et fournissant à la matière romanesque tel détail technique, telle date, tel fait, tel personnage périphérique. Si Aragon a souvent authentifié les paroles des personnages qu'il "cite", il laisse dans l'ombre les lectures qui alimentent les descriptions. Tout se passe comme si le discours déjà rapporté imposait l'authentification, alors qu'une scène ou une description, bien qu'elles empruntent à un texte, peuvent en faire l'économie; l'une comme l'autre répercutent pourtant un discours antérieur. Sans pouvoir ici multiplier les exemples, je mentionnerai seulement l'évocation du Portugal auquel rêve Toustaint. Ainsi à propos de son costume : "sous-lieutenant à la Caserne de Val de Freiro, habit rouge à revers, col et parements de velours noir, galons de laine blanche et boutons d'argent, le casque de feutre rouge à la crête de fourrure noire, et la cocarde blanche ${ }^{21}$ ".

Elle reprend presque mot à mot la description qu'en donne Toustaint dans ses Mémoires

Notre uniforme était rouge, revers, col et parements en velours noir, galons en laine blanche, boutons d'argent portant trois fleurs de lis, casque en feutre rouge avec crête en fourrure noire, cocarde blanche ; sur la plaque du baudrier : régiment de Mortemart ${ }^{22}$.

Quelques lignes plus loin, l'évocation de Lisbonne, de ses opéras et de ses hordes de chiens errants si elle procède du même texte, ne l'utilise plus que comme un canevas sur lequel il lui est loisible de broder :

Les Portugais, grands amateurs de musique, n'épargnent rien pour se procurer un spectacle, et leur Opéra est le plus beau de l'Europe. Ils ont engagé les deux plus célèbres artistes de l'Italie, le soprano Crescentini et Melle Catalini [...] Les représentations à son bénéfice donnaient plus de quarante mille francs; à l'une 
d'elles je l'ai vue recevoir, d'une femme de la cour, une aigrette de diamants de cinq mille cruzades [...] les chiens font courir les plus grands dangers aux personnes à cheval, dont ils épouvantent les montures en s'acharnant auprès d'elles d'une façon singulière, ce qui provoque de fâcheux accidents. [...] Les habitants de Lisbonne portent au milieu de la rue les animaux qui viennent à crever, chevaux, vaches, mulets, chats, etc. Ils servent de pâture aux chiens, exhalent une odeur effroyable et transforment la ville en une véritable voierie ${ }^{23}$. l'amplifie ; le passé revient ici hanter le sommeil de Toustaint sous le jour d'un baroque inquiétant : «Et à Lisbonne les bêtes mortes, les chats, les chevaux, les mulets exposés dans la rue, qu'une armée de chiens sauvages dévore en plein jour... des chiens, des milliers de chiens sur des charognes couvertes de mouches, des milliers de mouches... ${ }^{24}$ ».

21 Le caractère autographe du texte lu n'est pas déterminant puisqu'Aragon utilise de la même manière les biographies, en particulier, pour écrire la mort de Berthier, les pages que lui consacre le Général Derrécagaix ${ }^{25}$. Pour donner une idée de la variété des procédés de récriture que présente La Semaine sainte, mentionnons encore que les emprunts au texte lu sont parfois très limités ; ainsi dans ce passage :

Bon, il n'y avait pas que la Duchesse de Raguse... A l'époque de son mariage, Marmont avait plus à se faire pardonner ses origines aristocratiques qu'à les revendiquer. Son père, d'ailleurs, avant lui, avait pris pour femme une demoiselle de finances... Et c'était un général de Bonaparte que la fille du banquier Perregaux avait épousé, non pas ce nobliau de province, dont le blason n'était plus qu'un assignat ${ }^{26}$.

C'est à la biographie de Pierre Saint-Marc, Le maréchal Marmont, duc de Raguse, 1774-1852 qu'Aragon a emprunté l'information: «son père, Nicolas-Edme Viesse de Marmont était écuyer, seigneur de saint Colombe et du Moin-Monze, il épousa une fille de finances, Clothilde Hélène Victoire Chappron, née à Paris le 30 janvier 1710 » L'expression « fille de finances » constitue donc un emprunt littéral, qui indique par là même l'origine de la documentation; l'emprunt littéral permet de prouver la lecture antérieure qui a, dans une proportion indécidable, servi au portrait de Marmont ; mais dans l'ordre de la lecture, ce n'est pas le texte de La Semaine sainte qui indique le texte source (il n'y pas d'indice d'hétérogénéité).

Ces exemples appellent au moins deux remarques ; la première tient à la prudence que nécessite la lecture du roman d'Aragon ; l'usage de l'intertextualité y est si profus et si divers, et surtout, si savamment orchestré pour brouiller les cartes qu'il prétend abattre, qu'il est toujours risqué de se fier unilatéralement à ce que montre le texte ; apparemment explicitée, l'intertextualité peut dissimuler tout un pan caché de récriture. La seconde tient à sa spécificité dans le roman historique. Elle n'est pas uniquement liée à la nature des textes lus et récrits (mémoires, documents d'archive, journaux...) ; elle ressortit aussi au jeu qu'elle autorise entre fiction et invention, érudition et improvisation. Puiser la matière du roman dans le passé, c'est lire avant de l'écrire ; c'est donc faire de la récriture un enjeu majeur de la tension entre invention et érudition. Aragon avait sans doute beaucoup lu avant d'écrire son roman comme s'il le découvrait et le débat sur la sincérité de ses déclarations est vain ; ce qui importe, c'est de comprendre que la phrase, le phrasé doivent porter l'invention, lui impulser le rythme de l'improvisation, alors même que l'écriture s'adosse à de multiples lectures ; donner chair aux textes, c'est les prendre dans un rythme, celui de la vie, qui n'est pas celui de la lecture, dans un mouvement, celui de la progression chaotique et 
embourbée, qui n'est pas celui de l'archive, dans un désordre, qui n'est pas l'ordre des discours.

L'enquête intertextuelle, lorsqu'elle nous met aux prises avec ce type de matériaux, nous fait pénétrer dans l'atelier de l'écrivain; elle rejoint l'enquête généalogique au point de se confondre avec elle. Elle dessine l'utopie d'une lecture qui ferait les mêmes lectures que l'écrivain, qui, par cette coïncidence fantasmée des mémoires et des impressions, retracerait l'itinéraire de l'écriture. Mettre en évidence l'intertexte, dans le cas du roman historique, c'est donc retrouver le mélange de la fiction et de l'histoire, la manière dont l'une exploite les trésors, les lacunes, les faux semblants de l'autre. Elle permet aussi de corriger l'image d'une histoire mère nourricière du roman: si elle fournit des matériaux, la fiction ne s'inféode pas au discours de l'histoire ; La Semaine sainte récuse même toute idée de dépendance et de hiérarchie, donnant clairement la préférence au désordre et à l'anarchie. Loin d'établir une relation de filiation, le roman d'Aragon opère un vaste brassage (qui n'est pas une synthèse), un déplacement des codes et des genres. L'usage des textes lus, on l'a montré avec les quelques exemples choisis, n'est jamais systématique : les cartes sont à la fois montrées et cachées, le discours de l'histoire reconnu et nié, affiché et dissimulé, respecté et malmené, corrigé et autorisé. Et on aime à penser, qu'en amont, les lectures furent à la fois très nombreuses, très dispersées, toujours fécondes et jamais en pure perte. L'intérêt du roman historique à cet égard est immense, puisqu'il permet de confronter le texte littéraire et l'histoire qu'il récrit.

L'enquête intertextuelle, si elle donne des certitudes et des joies ponctuelles, nous entraîne aussi sur le seuil utopique d'une origine du roman. Un pas de plus, et c'est l'étude génétique, dont on sait la proximité avec l'approche intertextuelle. L'enquête généalogique et l'analyse génétique retournent au laboratoire secret de l'œuvre, à cette phase où écriture et lecture n'étaient peut-être pas si distinctes l'une de l'autre. La fascination pour l'intertextualité aurait alors à voir avec celle pour les archives, si frappante dans ce dernier quart du XXe siècle. Elle n'est sans doute pas indifférente à la passion de la généalogie. Mais alors, pourquoi ne pas intégrer dans le vaste giron de l'intertextualité tout ce qui a nourri le texte, aussi bien les images (et l'on sait l'importance de l'œuvre de Géricault dans ce roman) ou les propos oraux (on songe à l'entretien de la ferme des Ifs $^{27}$ ) ? Ils ont, comme les textes, nourri l'imagination, informé les représentations et impulsé l'écriture, lui donnant son rythme, sa tonalité, sa saveur aussi.

Si l'on s'y refuse, c'est que l'extension infinie de la notion lui fait perdre toute pertinence. D'abord parce qu'elle entraîne une dissolution aussi risquée de la notion de « texte » (le texte de la peinture, et pourquoi pas celui de la rumeur ou de l'enquête...); ensuite parce qu'elle disjoint la notion de la lecture. Enfin, elle risquerait de réduire l'intertextualité à un outil de l'étude généalogique ou génétique; or elle n'est pas une forme moderne de la critique des sources; si elle permet de retrouver ce qui précède le texte, elle conduit aussi à une approche rhétorique (c'est l'analyse de l'imitation ou de la transformation, proposée par Genette dans Palimpsestes), et intervient dans toute lecture herméneutique.

Revenons un instant, pour conclure, sur la spécificité de l'intertextualité dans La Semaine sainte, qui nous permet de mieux penser ce qui la distingue de la critique des sources. Celle-ci, cherche à réduire la multiplicité du texte lu pour obtenir un substrat sans résidu; le rêve serait le texte purifié de cette part de l'autre qui le constitue 
pourtant. La source, c'est ce qui irrigue le texte, le nourrit pour permettre son développement et son amplification. Trouver les sources, c'est trouver l'origine de l'origine. C'est donc régresser, réduire. La conception du texte sous-jacente est donc celle d'un noyau originel, que la prolifération de l'écriture ne doit pourtant pas dissimuler. La théorie du texte au contraire a valorisé la ramification infinie, la pulvérisation, la discontinuité, l'hétérogénéité (le texte comme mosaïque, puzzle, etc... ${ }^{28)}$. Le réseau, ou le rhizome, contredisent l'idée même d'origine. Ce que montre $L a$ Semaine sainte, c'est que l'écriture du roman procède par brassage et brouillage des textes, par refus de la hiérarchie des discours et de la séparation des pouvoirs respectifs de l'histoire et du roman; si une réflexion sur l'intertextualité fait pénétrer dans le laboratoire de La Semaine sainte, ce n'est en aucun cas pour délivrer la clé, l'origine de la création; c'est bien plutôt pour remettre en cause l'idée même d'origine, isolée et isolable. Comme le disait Jarry, l'écrivain n'assimile pas, il pulvérise: « un cerveau vraiment original fonctionne exactement comme l'estomac de l'autruche : tout lui est bon, il pulvérise des cailloux et tord des morceaux de fer. Qu'on ne confonde point ce phénomène avec la faculté d'assimilation, qui est d'une autre nature. Une personnalité ne s'assimile rien du tout, elle déforme ; mieux, elle transmute, dans le sens ascendant de la hiérarchie des métaux ${ }^{29}$ ".

C'est moins la graine qu'il faut retrouver, que l'éclat de la poudre.

\section{NOTES}

1. Voir par exemple « Secrets de fabrication ", J'abats mon jeu, E. F. R 1959 ; Je n'ai jamais appris à écrire ou les incipit, Skira, 1969, réédition Champs Flammarion, p. 102-109 et Henri Matisse, roman, Gallimard, 1971, tome II, «L'homme fait parenthèse ».

2. Claudie Bernard, Le Passé recomposé, le roman historique français du dix-neuvième siècle, Hachette supérieur, 1996.

3. Voir Henri Matisse, roman, tome II, p. 149.

4. Je me permets de renvoyer à mon étude "Les citations dans les romans d'Aragon ", actes du colloque Lire Aragon, 1997, Champion, 2000. et à la table des citations que j'ai fait paraître dans Recherches Croisées Aragon Triolet, $\mathrm{n}^{\circ} 6$.

5. La Semaine sainte, Gallimard, 1958, « Folio », 1998, p. 612- 614. Voir p. 407-408;

520-521 ; 532. Rappelons que la traduction de Sevelinges est parue en 1803.

6. La Semaine sainte, p. 596.

7. Ibid., p. 452 et 453.

8. Le texte extrait de Les Communistes, Messidor, tome 2, p. 482. Il est remarquable qu'Aragon décale la date : dans les Communistes, il s'agit de «cet interminable 28 mai », alors que dans La Semaine sainte on lit : « Dans la nuit du 26 au 27 mai 1940, après avoir traversé Armentières en feu, dans nos voitures assaillies par une chaleur suffocante et des brindilles incandescentes, des flammèches qui entraient par la portière, nous avions débarqué ici, des éléments de la $3^{\mathrm{e}} \mathrm{D}$. L. M. Un avion qui avait bombardé les bâtiments avait été abattu non loin de la ferme. J'ai décrit cela quelque part, pourquoi 
recommencer [suit la citation des Communistes] ». La Semaine sainte, p. 795 ; c'est moi qui souligne. On peut à ce sujet émettre l'hypothèse qu'Aragon corrige ici le roman, comme il corrige ailleurs le discours de l'histoire.

9. La Semaine sainte, p. 796.

10. Ibid., p. 813.

11. Ibid., p. 737-738.

12. Voir ibid., p. 766-767.

13. Pour les modifications du texte de Lamartine, nous renvoyons au livre de Patricia Principalli, La Semaine sainte d'Aragon, Un roman du « passage », L'Harmattan, 2000, p. 57.

14. Ibid., p. 819-820. En italiques, la citation s'étend sur une quinzaine de lignes ; Aragon l'emprunte à Antonin Debidour, Le Général Fabvier, sa vie militaire et politique, Plon et Nourrit, 1904, p. 234-235 et 238.

15. La Semaine sainte, p. 115-116; voir d'une part, Jean de La Tour, Duroc, duc de Frioul, grand maréchal du palais impérial, 1772-1813, Librairie militaire Chapelot, 1913, p. 302 qui cite les mémoires de Caulaincourt, rapportant le mot de Duroc, et d'autre part, Mémoires de la duchesse d'Abrantès, Jean de Bonnot, 1968, p. 206-207, tome 11.

16. Voir en particulier « Secrets de fabrication », op. cit., p. 50-56 et Edouard Béguin, "La genèse de Simon Richard d'après le dossier manuscrit de la Semaine sainte, naissance du personnage, naissance du roman », Histoire/Roman, La Semaine sainte, Publications Université de Provence, 1988.

17. La Semaine sainte, p. 675.

18. Voir La Police secrète du premier Empire, librairie Perrin, 1908, préface de Louis Madelin. L'anecdote est une amplification de la phrase suivante : «Son père signale une maison où la nuit on entend du bruit, comme si on gardait quelqu'un : c'est une

boulangerie ». (Bulletin du 14 fructidor an XII, samedi $1{ }^{\mathrm{er}}$ septembre 1804, p. 70 ; c'est moi qui souligne). Dans les « Secrets de fabrication », Aragon commente longuement l'invention de ce personnage et mentionne les papiers publiés par Louis Madelin («Secrets de fabrication », op. cit., p. 50-56).

19. Voir respectivement La Semaine sainte, p. 196 et p. 659. Chateaubriand relate dans les Mémoires d'outre-tombe le départ du roi le 20 mars, ainsi que sa propre fuite à Gand, en voiture avec Madame de Chateaubriand, via Amiens et Arras (Mémoires d'outre-tombe, Gallimard, «Bibliothèque de la pléiade », 1951, tome I, p. 926-927) ; Aragon récrit, avec ironie, Laurette ou le cachet rouge, Souvenirs de servitude militaire, Garnier Frères, 1965, p. 29-30.

20. Pour de plus amples exemples, je me permets de renvoyer à l'édition de La Semaine sainte dans la Bibliothèque de la Pléiade, que j'établis pour Gallimard (à paraître).

21. La Semaine sainte, p. 282-283.

22. Mémoires du marquis de Toustaint, publiés par la marquise de Perry de Nieuil, Plon, 1933, p. 29.

23. Ibid.

24. La Semaine sainte, p. 284.

25. Voir Ibid., p. 623 sq. Aragon procède en amplifiant le récit de Derrécagaix, Le Maréchal Berthier, prince de Wagram et de Neuchâtel, librairie militaire R. Chapelot et compagnie, 1904, p. 600.

26. La Semaine sainte, p. 315.

27. Voir « Secrets de fabrication », op. cit., p. 68.

28. Voir en particulier Julia Kristeva, Séméiotikè, Le Seuil, 1969 ; Roland Barthes «Théorie du texte » Encyclopédie Universalis, 1973 et Gilles Deleuze, Félix Guattari, Mille 
plateaux : « Un premier type de livre, c'est le livre-racine. L'arbre est déjà l'image du monde, ou bien la racine est l'image de l'arbre monde. C'est le livre classique, comme belle intériorité organique, signifiante et subjective (les strates du livre) [...] Le système radicelle ou racine fasciculée, est la seconde figure du livre, dont notre modernité se réclame volontiers. Cette fois, la racine principale a avorté, ou se détruit vers son extrémité ; vient se greffer sur elle une multiplicité immédiate et quelconque de racines secondaires qui prennent un grand développement. [...] ». Minuit, 1980, p. 1-12.

29. Alfred Jarry, «La Chandelle verte », CEuvres Complètes, tome II, p. 393, Gallimard, «Bibliothèque de la Pléiade ».

\section{RÉSUMÉS}

Roman historique, La Semaine sainte repose sur une documentation érudite très importante. Elle donne lieu à une intertextualité massive, qui prend souvent la forme de la citation explicite, dont la fonction est de consolider l'exactitude historique de la fiction. L'amplitude des emprunts peut être très variée, et seule une enquête érudite et minutieuse permet de retrouver les fragments montés dans le roman. Aragon ne révèle en effet qu'une partie infime des intertextes qu'il exploite pour écrire La Semaine sainte; sous une forme implicite, l'intertextualité établit une relation polémique entre l'histoire et le roman. L'invention romanesque s'alimente en effet au discours historique qu'elle n'utilise pas aux seules fins de véracité, mais aussi pour le corriger ou le démentir. Jamais systématique, l'usage de l'intertextualité dans La Semaine sainte montre comment le roman historique procède en brouillant les cartes de l'histoire et de la fiction.

\section{AUTEUR}

\section{NATHALIE PIÉGAY-GROS}

Université Paris 7-Denis Diderot 